Preprints of the

Max Planck Institute for

Research on Collective Goods

Bonn 2013/8

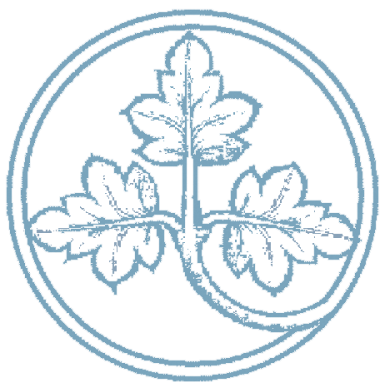

Wondering How Others Interpret It: Social Value of Public Information

Alia Gizatulina

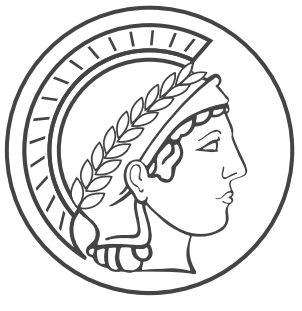




\section{Wondering How Others Interpret It: Social Value of Public Information}

Alia Gizatulina

April 2013 


\title{
Wondering How Others Interpret It: Social Value of Public Information*
}

\author{
Alia Gizatulina ${ }^{\dagger}$ \\ This version: April 2013
}

\begin{abstract}
This paper studies the social value of public information in environments without common knowledge of the data-generating process. We show that the stronger the coordination motive behind agents' behaviour is, the more they use private or public signals in the way that they suspect others are doing it. Consequently, the negative impact of public communication noted by Morris and Shin (2002) can be amplified if agents suspect that others take the public signal too literally and/or are too inattentive to their private signals. Social welfare, if measured as in Morris and Shin (2002), always increases in the precision of the public signal when each agent evaluates its precision correctly, but believes that others did not understand the public signal at all, which suggests that there is a scope to "obliterate" public communication in a specific way, by making it, e.g., sophisticated and technical. By contrast, measuring welfare as in Woodford (2005) reverses, in general, desirability for such obliteration and non-commonality of signals' understanding.
\end{abstract}

Keywords: central bank communication, transparency, common p-belief, coordination game, higher-order uncertainty

JEL: D82, E58

*This paper has circulated earlier under the title "Disagreement and Social Value of Public Information". I am very grateful for helpful comments to Olga Gorelkina, Christoph Engel, Martin Hellwig, Wolfgang Kuhle, Nicolas Roux, Karl Schlag and audiences of the EEA-ESEM 2011 Meeting and the CESifo Workshop on Applied Microeconomics.

${ }^{\dagger}$ Max Planck Institute for Research on Collective Goods, Bonn, GERMANY. Email: gizatulina@coll.mpg.de 


\section{Introduction}

Should the government or any other central authority reveal publicly all its available information relevant for agents' efficient decision-making? There have been several suggestions in the literature for the optimal degree of transparency that a central authority, providing information about economic fundamentals, should adopt in order to maximize social welfare in environments where agents play the beauty-contest type of games. Agents use this information in order to update their expectations about the payoff-relevant fundamentals. They also use this information in order to predict the behaviour of others and to coordinate better with others' actions. Thus it would seem that more of available information is desirable.

In their seminal paper, Morris and Shin (2002) took issue with this view and argued that if agents' utility depends to a large extent on coordination with each other (rather than on how their actions match fundamental economic values) the government (or, in their case, the central bank) should be transparent only if it is able to provide sufficiently precise public information, specifically more precise information than the information that agents possess themselves. Those predictions are strengthened when importance of coordination motives increases as compared to importance of fundamentals in the agents' utility function. In contrast, Hellwig (2005), in a richer setup, though with a more specific structure of fundamentals and the welfare measure, argued that an increase of precision of public information is always beneficial. Later Angeletos and Pavan (2007) generalized the framework of Morris and Shin (2002) and within the cases of their analysis as in the setup of Morris and Shin (2002) they reconfirm the prescriptions of Morris and Shin (2002) on the optimal degree of the precision of public information 1 .

Those papers, while different in the assumptions on utilities and relevant welfare measures, were similar in their assumption about the way information affects agents' beliefs. Specifically, in those models, agents agree on how to interpret the public and private signals and this agreement is common knowledge among

\footnotetext{
${ }^{1}$ Other papers which studied the same question are Cornand and Heinemann (2008), Morris and Shin (2007) and Myatt and Wallace (2009). All those papers came essentially to the same conclusion, social welfare can be decreasing in the precision of the common signal, however the optimal policy should try to reduce the commonality of the signal as much as possible rather than the precision per se.
} 
agents. The only source for difference in beliefs was privately known individual signals, i.e., agents disagree only because of differences in their private information, and had private signals been commonly exchanged there would be no disagreement (and it would be always beneficial to increase the precision of public information).

The assumption of common knowledge and agreement among agents on the data-generating process has been prevalent in the theoretical literature for decades. Under this assumption, agents, exposed the same evidence, cannot disagree on how signals should be interpreted or they cannot agree to disagree about the expected value of the unknown variable. Furthermore, even if agents are exposed to different, privately known facts, but they know each other's posterior expectations of a given random variable, they still cannot agree to disagree about the expected value of this random variable (see, e.g., Morris (1995)). Given this fact, agents cannot behave differently exposed to the same information while having the same preferences. In the meanwhile, however, the empirical and experimental literature has documented a vast amount of robust evidence which is inconsistent with those statements and hence with the assumption of common knowledge and agreement on the data-generating process.

One of the most robust observed phenomenon of misjudgment and departure from a rational take of facts is overconfidence. More often than not agents suffer from overconfidence, be it absolute overconfidence (they believe to perform better than they actually do) or relative overconfidence (they believe their performance to be better than the performance of their peers). Such overconfidence occurs with regard to the ability to perform physical tasks, but also during decision-making and interpretation of payoff-relevant information. As an example of relative overconfidence, Montier (2006) documents how out of 300 professional fund managers $74 \%$ believed that they had delivered above average performance in recent years, and the remaining $26 \%$ believed that they were just as good as the average fund manager. A study by Svenson (1981) shows how, in a sample of U.S. students, $82 \%$ of them believe they are in the top $30 \%$ of the group who is driving most safely ${ }^{2}$. As an example of absolute overconfidence,

\footnotetext{
${ }^{2}$ See also the discussion in Benoit and Dubra (2011) that such (relative) overconfidence may be only apparent and can be justified by absence of opposite evidence about own skills. Burks, Carpenter, Goette, and Rustichini (2013), on the other hand, show in an experimental study that the "apparent overconfidence" of Benoit and Dubra (2011) is not that apparent - agents' selfconfidence in their experiments is inconsistent with Bayesian decision making.
} 
Ben-David, Graham, and Harvey (2010) show that top financial executives are severely miscalibrated when asked to predict future returns, the eventually realized market returns fall only $33 \%$ of times into their $80 \%$ confidence interval, i.e., financial executives are persistently failing to learn to make correct inferences from the observed data and kept on believing in own models of the world.

Another instance of misjudgment, related to overconfidence, appears in abnormal trading volumes after quarterly announcements of corporate earnings, as found in Kandel and Pearson (1995) ${ }^{3}$. The volumes of documented trade cannot be explained by a model where all agents are interpreting the same public signal in the same way, even if they possess some private information. For the problem of macroeconomic forecasting, Lahiri and Sheng (2008) provide a largescale study on how professional forecasters of macro variables, while arguably observing roughly the same statistical data, persistently disagree on the future rates of inflation, unemployment and GDP growth. As they revise and publish anew their forecasts regularly and, plausibly, have occasionally a look at what their peers have forecasted, if they shared the common prior and the same model of the world, their next forecasts would be the same at a faster rate than what is observed.

Furthermore, it is not uncommon for arguably sophisticated agents to commonly agree to disagree. Such public disagreement is impossible under the common prior assumption. E.g., Odean (1998) provides an example how Warren Buffet and the Feshbach brothers held, respectively, long and short positions worth hundreds of millions of dollars in Wells Fargo Bank and while the bank's weak loan portfolio was public information, investors had quite different models on the impact that this could have on its future value. This difference in opinion was common knowledge between them, yet it did not seem to induce them to question their models of the world (in the end the Feshbachs lost \$50 million when closing their positions). A similar, more recent example in this vein is the short position of James Chanos with regard to securities linked to China's economy. Chanos, while accusing China of artificially creating growth statistics and overall bubbleness, makes large bets on the imminent collapse of China's economy. Many other large financial market players are have a completely opposite

\footnotetext{
${ }^{3}$ See also the result in Glaser and Weber (2007) who show that overreaction to public announcements and abnormal trading documented in the literature are positive correlated with overconfidence but not with miscalibration.
} 
opinion (including e.g. Warren Buffet, Jim Rogers and Wilbur Ross)

Empirical evidence that people polarize and diverge in their opinions even more once they have observed the same (public) information is another case supporting absence of common knowledge and agreement on the data-generating process. For example, Lord, Ross, and Lepper (1979) documented an experiment where two groups of subjects supporting and opposing the death penalty were exposed to two purported studies, one seemingly confirming and one seemingly disconfirming their beliefs about the efficacy of the death penalty in deterring criminal behaviour. Both proponents and opponents of the death penalty considered the results and procedures that confirmed their own beliefs to be more convincing and probative ones, so they shifted beliefs even further to the extremes. Another example is Batson (1975), who shows how religious believers disagree even more with non-believers after having been presented with a "study" that the New Testament is a fake.

In light of those observations, it seems necessary to evaluate the question of optimal degree of transparency in environments where agents do not "commonly agree to agree" on the data-generating process ${ }^{5}$. As the above empirical findings suggest, there is a multitude of patterns how agents beliefs can fail to be consistent with the assumption that agents commonly agree on the data-generating process (i.e., they have a common prior).

In this paper, we follow a relatively conservative way to relax this assumption. We consider a class of environments where agents, in fact, agree on the data-generating process but they do not know it commonly. This is the smallest possible departure from the assumption of existence of a commonly known and agreed upon prior. Yet, as we will show, it can bring significantly different conclusions for optimal communication as compared to the early results in the literature.

To introduce absence of common knowledge of the data-generating process, we consider the environment where doubts about others' interpretation of public and private signals take the form of a (common) $p$-belief. Each agent believes

\footnotetext{
4See the article in The New York Times "Contrarian Investor Sees Economic Crash in China" from January 7, 2010

${ }^{5}$ As far as interpretation of public signals sent by the central bank is concerned, Rosa and Verga (2008) show that financial market participants need around 3 years to learn (commonly) how to interpret and believe the ECB announcements of its policy plan - something which is not allowed by the previous literature on optimal communication by the central bank.
} 
that it is only with probability $p$ that others have the same model of the joint stochastic evolution of fundamentals and private and public signals, and with probability $(1-p)$ they interpret a given private or public signal as being actually of a different precision. Furthermore, each agent believes that others have the same doubts as he has about everyone's interpretation only with probability $p$ and with probability $(1-p)$ others are certain that everyone else is certain that precisions of signals are different from what is believed with probability $p$. And so on. In the Section 5 we also discuss results under a stronger assumption when agents agree to disagree, i.e., agents commonly know and agree that they do not share a common prior.

As the main results of the paper, we decompose the effect of absence of common knowledge of the objective data-generating process along of two layers. First we study the value of more precise public information in environments where each agent suspects that with probability $(1-p)$ others fail to have the right precision of public signals. In the second case, each agent suspects that others may fail to have the right interpretation of their private signals.

We find that the effect of more transparent communication on individual actions, and hence total welfare, depends on fine details of each environment. It matters whether agents believe with probability $(1-p)$ that others fail to have the correct interpretation by overreacting to public (or private) signals, i.e., others take those signals too literally or by underreacting to it, i.e., others just miss paying enough attention to the valuable signals. Thus, in our framework, agents are relatively overconfident in the sense that they suspect others to be less sophisticated and naive by taking the signals at face value or not noticing them enough, but we do not fix either of those possibilities and study both cases.

We further decompose the effect of public communication by considering the possibility that "common $p$-believing" is partially endogenous to public communication, i.e., a public signal has a "multidimensional effect" on agents' beliefs, potentially different for different orders of beliefs. Partial endogeneity means that we assume that $p$ is fixed but the precision which is believed with probabilities $p$ and $(1-p)$ is endogenous to the structure of public communication. Such structure is rich enough to include the standard case where the data-generating process is commonly known (e.g., when what is $p$-believed and what is $(1-p)$ believed is the same).

For the welfare measure as in Morris and Shin (2002), our findings are as follows: if agents believe that with probability $(1-p)$ everyone is overreacting to 
the public signal or everyone is underreacting to his private signals, and if $(1-p)$ is significantly high, the communication can be even more detrimental to social welfare than it is in Morris and Shin (2002). On the other hand, if agents believe that with probability $(1-p)$ everyone is underreacting to the public signal or everyone is overreacting to his individual signals, the negative effect of increased transparency of Morris and Shin (2002) disappears. The reason for those results is that each agent, despite having the correct evaluation of relative importance of signals, overreacts in his actions to public information (or underreacts to his private signals) if he suspects others to do so. That is, paradoxically, the more the agent is confident (correctly) about own abilities to interpret the signals relative to his beliefs about others abilities, i.e., the more he believes others to be incorrect, the more he forgoes his correct estimation and uses the signals like he believes others are using them. Thus, extending the conclusions of Hellwig and Veldkamp (2009), agents not only would like to know what others know, but because of coordination motives, they also would like to follow the signals in a way that they believe others are interpreting them, even if doing it contradicts their own model of the world.

Taking into account the scope for public communication to have a separate impact on what is commonly $p$-believed from the impact on what is, roughly, $(1-p)$-believed, our conclusions for the optimal degree of transparency are that public communication should be as clear as possible from the perspective of each agent's own interpretation of the public signal and it should be as "muddy" as possible to induce each agent to believe that others did not understand as much from his communication as this agent himself did. For example, public communication can be very technical, i.e., very precise, on the one hand, but also inducing doubts in each agent whether this (technical) signal has been really informative for everyone else. Reconsidering communication by a central bank, the much-quoted statement of Alan Greenspan's that "...I guess I should warn you, if I turn out to be particularly clear, you've probably misunderstood what I said.." can have clear welfare-increasing effects, provided it reinforces each agent's selfconfidence in his (correct) model of the world, but also makes him believe that it diluted the amount of information in the central bank's message as perceived by other agents.

Next we consider the case where agents commonly agree to disagree about the ex ante expected value of the fundamental. The robust result in this case is that greater transparency outweighs losses due to miscoordination among agents 
arising because of disagreement about the data-generating process. Thus, assuming that in reality there is a mixture of doubts about others' interpretation capacity and agreement to disagree, the optimal communication policy should take into account both effects of increased transparency.

We also consider a different welfare measure, accounting for losses due to agents' miscoordination with each other, following the suggestion in Woodford (2005). Contrary to his result, obtained under common knowledge and agreement about the data-generation process and stating that under this different welfare measure greater transparency is always valuable, under common $p$-belief about the prior, with $p<1$, the detrimental effect of public communication can appear again. The reason for this is at high $r$ and suspicion that others are overreacting to the public signal (i.e. they take it as too precise) welfare gains from better coordination with each other, due to more value put on the same public signal, are lower than losses due to more value put on the (likely) wrong public signal.

Another surprising result for this welfare measure when $p<1$ is the possibility for welfare's detrioriation due to too noisy private signals - an effect which is never available under the welfare measure in Morris and Shin (2002) or in Woodford (2005) with $p=1$. This effect is available both when agents have doubts about others' interpretation of public and also of private signals and when $r$ is sufficiently high. The reason is that too imprecise private signals distract agents from efficient coordinating with each other and in some constellations welfare is higher when there is no any private information at all (if it cannot be more precise than a certain threshold).

Finally, if the relevant welfare measure is as in Woodford (2005), the previous prescription to introduce noise into public communication provided it induces agents to believe that others do not pay attention to public signals, is not valid anymore. Especially if the precision of public signal can be made higher than the precision of private signals. In this case, communication should be also as clear as possible in order to induce the strongest possible common understanding of public signals. 


\section{The Model}

The basic assumption about the utility function of agents is the same as in Morris and Shin (2002). There is a continuum of agents of mass 1 , indexed by $i \in[0,1]$. Each agent's utility is

$$
u_{i}(a, \theta)=-(1-r)\left(a_{i}-\theta\right)^{2}-r\left(L_{i}-\int_{0}^{1} L_{j} d j\right)
$$

with $r$ constant and $0<r<1$. The parameter $\theta$ stands for a fundamental, $a_{i}$ is action taken by agent $i$. The term $L_{i} \equiv \int_{0}^{1}\left(a_{j}-a_{i}\right)^{2} d j$ is the beauty contest term, a penalty that agents incur for deviating from actions of others.

The value of $\theta$ is unknown and represents, from the perspective of agents, a random variable. Each agent has some probabilistic assessments of $\theta$ and also beliefs about others' assessments. We will introduce more details below.

Given the individual utilities, we can now state the social welfare function (which, for the moment, is the same as in Morris and Shin (2002) and we will discuss in the later section how predictions of the analysis change if the relevant welfare assessment taken as in Woodford (2005)).

$$
\begin{aligned}
W(a, \theta) & =\frac{1}{1-r} \int_{0}^{1} u_{i}(a, \theta) d i \\
& =-\int_{0}^{1}\left(a_{i}-\theta\right)^{2} d i
\end{aligned}
$$

Hence, from the aggregate perspective it is desirable that each agent's action matches perfectly $\theta$.

\section{Some preliminary analysis}

From the agent's maximization program we can derive agent's best reply function

$$
a_{i}\left(t_{i}\right)=(1-r) E_{i}\left[\theta \mid t_{i}\right]+r E_{i}\left[\bar{a} \mid t_{i}\right]
$$

where $\bar{a}$ is the average action of other agents.

It is useful to note that if $\theta$ were commonly known, the (unique) optimal action would be

$$
a_{i}^{*}=\theta
$$


which coincides with the action maximizing social welfare in (2). That is, under complete information there is no distortions due to miscoordination and social welfare is at its maximum.

When agents do not possess perfect knowledge of the value of $\theta$, their action depends on their probabilistic beliefs about $\theta$; furthermore, to find the optimal best reply to others' action they need to evaluate what other agents' beliefs are. Similarly agents possess third and higher orders of beliefs about everyone else belief about... $\theta$. Thus, each order of beliefs has an impact on the equilibrium strategy $a_{i}^{*}$. By iterative evaluation of the best reply function we obtain a solution to agents' maximization problem in terms of their higher order beliefs as follows 6

$$
a_{i}^{*}\left(t_{i}\right)=(1-r) \sum_{k=1}^{\infty} r^{k} E_{i}\left(\bar{E}^{k}(\theta)\right)
$$

where $E_{i}\left(\bar{E}^{k}(\theta)\right)$ means the expectation of agent $i$ of the average expectation of the average expectation, etc. $k$ times (e.g. $\left.E_{i}\left(\bar{E}^{2}(\theta)\right)=E_{i}[\bar{E}[\bar{E}[\theta]]]\right)$. Thus, the optimal action in (5) depends only on the first moment of each order of expectations about $\theta$. In the next section we introduce the details of $\left\{E_{i}\left(\bar{E}^{k}(\theta)\right)\right\}_{k=1}^{\infty}$ without common knowledge of the data-generation process.

\section{Common $p$-Belief About the Data-Generating Pro- cess}

\subsection{Interim Beliefs}

Following the literature we let the (objective) distribution of the fundamental be Gaussian with parameters $\mu$ and $\alpha$ :

$$
\theta \backsim \mathcal{N}\left(\mu, \frac{1}{\alpha}\right)
$$

Whereas agents commonly know and agree that the fundamental follows the distribution from the Gaussian family, they are uncertain which specific values parameters $\mu$ and $\alpha$ assume (the details of this uncertainty are spelled out below).

\footnotetext{
${ }^{6}$ This has been also derived in Morris and Shin (2002) (expression (14) on p.1526)
} 
We assume that it is common knowledge that signals are linear in $\theta$. The public signal is given by

$$
y=\theta+\eta
$$

where the distribution of $\eta$ is from the family of normal distributions

$$
\eta \backsim \mathcal{N}\left(0, \frac{1}{\beta}\right)
$$

and again agents may possess different and only privately known beliefs about the parameter $\beta$.

In addition to the public signal agents observe individual signals

$$
x_{i}=\theta+\varepsilon_{i}
$$

where the noise $\varepsilon_{i}$ is distributed according to

$$
\varepsilon_{i} \backsim \mathcal{N}\left(0, \frac{1}{\gamma}\right) .
$$

In Morris and Shin (2002) and the subsequent related literature the values of the parameters $\alpha, \beta, \gamma, \mu$ are assumed to be the same and commonly known across all agents. To depart from this assumption and introduce absence of common knowledge about parameters $(\alpha, \beta, \gamma, \mu)$ of the (objective) distribution of the vector $(\theta, y, x)$ we follow the framework of Monderer and Samet (1989) and allow agents to possess common $p$-beliefs about the stochastic relationship between fundamentals and signals.

The common $p$-belief about the prior joint distribution is defined here by the following sequence of iterative statements:

1. Every agent believes with probability one that the joint distribution of $(\theta, y, x)$ is given by $\mathcal{N}(\mu, \Sigma(\alpha, \beta, \gamma))$

2. Every agent believes that [1] holds with probability $p$ and that with probability $(1-p)$ everyone believes that with probability 1 the joint distribution of $(\theta, y, x)$ is given by some $\mathcal{N}\left(\boldsymbol{\mu}^{\prime}, \Sigma^{\prime}\right)$

3. Every agent believes that [2] holds with probability $p$ and that with probability $(1-p)$ that everyone believes that with probability 1 that everyone believes that with probability 1 the joint distribution of $(\theta, y, x)$ is given by $\mathcal{N}\left(\mu^{\prime}, \Sigma^{\prime}\right)$. 
4. .....

Thus each agent knows himself perfectly well the actual data-generating process. But at each order each agent suspects that with probability $(1-p)$ everyone else believes $(\theta, y, x)$ be drawn from $\mathcal{N}\left(\mu^{\prime}, \Sigma^{\prime}\right)$ and moreover that everyone is certain that everyone else is certain that the distribution is $\mathcal{N}\left(\boldsymbol{\mu}^{\prime}, \Sigma^{\prime}\right)$. Note that here as $p \rightarrow 1$ the environment above converges to the environment where $\mathcal{N}(\mu, \Sigma(\alpha, \beta, \gamma))$ is commonly known, like it is in Morris and Shin (2002), which is, thus, a special case of our framework. On the other hand, if we let $p \rightarrow 0$ agents agree to disagree (though we need to modify distributions $\mathcal{N}(\mu, \Sigma)$ and $\mathcal{N}\left(\boldsymbol{\mu}^{\prime}, \Sigma^{\prime}\right)$ to be consistent in the limit).

In the construction above, at the first order, each agent actually assesses correctly that the joint distribution of $(\theta, y, x)$ is given by $\mathcal{N}(\boldsymbol{\mu}, \Sigma(\alpha, \beta, \gamma))$. Instead, of course, one could have introduced doubts about $\mathcal{N}(\mu, \Sigma(\alpha, \beta, \gamma))$ already at the first order. But as we would like first to distill out the effect of absence of common knowledge rather than the effect of absence of individual knowledge, i.e. that already at the first level each agent is uncertain what the data-generating process is, we follow the route above.

It is also noticeable that there are several different ways to relax the common knowledge assumption in this framework. Rather than considering a two-points uncertainty as here, we could have introduced a richer structure of doubts and let, for example, that each agent believes with probability $\left(1-p_{1}\right)$ everyone believes that the relevant distribution is $\mathcal{N}\left(\boldsymbol{\mu}^{\prime}, \Sigma^{\prime}\right)$ and with probability $\left(1-p_{2}\right)$ everyone believes that the distribution is some $\mathcal{N}\left(\boldsymbol{\mu}^{\prime \prime}, \Sigma^{\prime \prime}\right)$, with $p_{1}+p_{2}=p+1$. The higher levels of uncertainty can be enriched further along the same lines. Whereas such extensions are interesting, as we will discuss later in more details, the results cannot be expected to be qualitatively much different from the current framework.

\subsection{Equilibrium}

To disentangle different effects of absence of common knowledge of the datageneration process we will consider separately the case where agents have doubts about others' assessment of the actual precision of the public signal, i.e. of $\beta$ and the case where agents have doubts about others' interpretation of their private 
signals 7

\section{Absence of common knowledge of $\beta$}

We first consider the case when agents are not certain how others interpret the public signal. Specifically the higher orders of beliefs of each agent are

1. Every agent believes that with probability one the precision of the public signal is equal to $\beta$

2. Every agent believes that [1] holds with probability $p$ and that with probability $(1-p)$ everyone believes that with probability 1 the precision of the public signal is $\beta^{\prime}$.

3. Every agent believes that [2] holds with probability $p$ and that with probability $(1-p)$ everyone believes that with probability 1 everyone believes that with probability 1 the precision is $\beta^{\prime}$.

4. .....

The usual technique to solve the equilibrium by the method of undetermined coefficients cannot be applied to our framework, because agents' second (and higher) order of beliefs are totally independent of their first order beliefs. The method of undetermined coefficients uses only the first order of expectations and relies implicitly on the fact that all information about the belief hierarchy can be fully derived from $\left\{E_{i}\left[\theta \mid y, x_{i}\right]\right\}_{i=1}^{I}$ or more specifically from the common prior, fully expressed in $\left\{E_{i}\left[\theta \mid y, x_{i}\right]\right\}_{i=1}^{I}$ (which is common knowledge) and the fact that all variables are normally distributed.

Hence, as the equilibrium strategy can be expressed as a weighted sum of iterated average expectations (see the expression (5)), to calculate it we will construct the infinite sequence of iterated expectations resulting from the iterative $p$-beliefs reasoning above, substitute it into the expression (5) and simplify. The first order of expectations is equal to

$$
E_{i}\left[\theta \mid y, x_{i}\right]=\frac{\alpha \mu+\beta y+\gamma x_{i}}{\alpha+\beta+\gamma}
$$

\footnotetext{
${ }^{7}$ In the Appendix A.2 we argue that considering effect of absence of common knowledge of precisions of public and private signals is without much loss of generality, as two effects enter in an "additive" manner into the equilibrium strategy.
} 
the second order expectation, i.e. expectation about the average of others' expectations is

$$
\begin{gathered}
E_{i} \bar{E}\left[\theta \mid y, x_{i}\right]=p\left(\frac{\alpha \mu+\beta y}{\alpha+\beta+\gamma}+\frac{\gamma}{\alpha+\beta+\gamma}\left(\frac{\alpha \mu+\beta y+\gamma x_{i}}{\alpha+\beta+\gamma}\right)\right)+ \\
\quad+(1-p)\left(\frac{\alpha \mu+\beta^{\prime} y}{\alpha+\beta^{\prime}+\gamma}+\frac{\gamma}{\alpha+\beta^{\prime}+\gamma}\left(\frac{\alpha \mu+\beta y+\gamma x_{i}}{\alpha+\beta+\gamma}\right)\right)
\end{gathered}
$$

the third order expectations are then

$$
\begin{gathered}
E_{i} \bar{E} \bar{E}\left[\theta \mid y, x_{i}\right]=p\left[p\left(\frac{\alpha \mu+\beta y}{\alpha+\beta+\gamma}+\frac{\gamma}{\alpha+\beta+\gamma}\left(\frac{\alpha \mu+\beta y+\gamma x_{i}}{\alpha+\beta+\gamma}\right)\right)+\right. \\
\left.+(1-p)\left(\frac{\alpha \mu+\beta^{\prime} y}{\alpha+\beta^{\prime}+\gamma}+\frac{\gamma}{\alpha+\beta^{\prime}+\gamma}\left(\frac{\alpha \mu+\beta y+\gamma x_{i}}{\alpha+\beta+\gamma}\right)\right)\right]+ \\
+(1-p)\left[\frac{\alpha \mu+\beta^{\prime} y}{\alpha+\beta^{\prime}+\gamma}+\frac{\gamma}{\alpha+\beta^{\prime}+\gamma}\left(\frac{\alpha \mu+\beta^{\prime} y}{\alpha+\beta^{\prime}+\gamma}+\frac{\gamma}{\alpha+\beta^{\prime}+\gamma}\left(\frac{\alpha \mu+\beta y+\gamma x_{i}}{\alpha+\beta+\gamma}\right)\right)\right]
\end{gathered}
$$

One can derive similarly the forth and subsequent orders of expectations. To simplify the exposition somewhat we introduce the following notation

$$
\begin{aligned}
A & :=\frac{\alpha \mu+\beta y}{\alpha+\beta+\gamma} \\
B & :=\frac{\alpha \mu+\beta y+\gamma x_{i}}{\alpha+\beta+\gamma} \\
C & :=\frac{\alpha \mu+\beta^{\prime} y}{\alpha+\beta^{\prime}+\gamma} \\
k & :=\frac{\gamma}{\alpha+\beta+\gamma} \\
q & :=\frac{\gamma}{\alpha+\beta^{\prime}+\gamma}
\end{aligned}
$$

Using this notation and substituting $\left(E_{i}\left[\theta \mid y, x_{i}\right], E_{i} \bar{E}\left[\theta \mid y, x_{i}\right], \ldots\right)$ into (5) we obtain the equilibrium strategy as the sum of series 


$$
\begin{gathered}
a_{i}=(1-r) B+(1-r) r(p(A+k B)+(1-p)(C+q B)) \\
+(1-r)(1-p) \sum_{s=2}^{\infty} r^{s}\left\{C\left[\sum_{m=0}^{s-1} q^{m}+\sum_{m=0}^{s-2} p^{m+1} \sum_{n=0}^{s-m-2} q^{n}\right]\right. \\
+A\left[p^{s} \sum_{m=0}^{s-1} k+\sum_{m=0}^{s-2} p^{m+1} q^{s-m-1} \sum_{j=0}^{m} q^{j}\right] \\
\left.+B\left[q^{s}+p^{s} k^{s}+\sum_{m=0}^{s-2} p^{s-m-1} q^{m+1} k^{s-m-1}\right]\right\}
\end{gathered}
$$

Simplifying the summands we obtain

$$
a^{*}=\frac{(1-r) r p(1-p q r) A}{(1-p r)(1-q r)(1-k p r)}+\frac{(1-r)(1-p q r) B}{(1-k p r)(1-q r)}+\frac{r(1-p) C}{(1-p r)(1-q r)}
$$

To make our results more directly comparable to Morris and Shin (2002) we let $\alpha=0$ for the moment, i.e. the fundamental $\theta$ has the improper prior (with infinite prior variance). Using this and (9) we simplify (11) to obtain

$$
a_{i}^{*}\left(y, x_{i}\right)=\frac{\beta^{\prime} \gamma r(1-p)+\beta\left(\beta^{\prime}+\gamma(1-r)\right)}{\left(\beta^{\prime}+\gamma(1-r)\right)(\beta+\gamma(1-p r))} y+\frac{\left.\beta^{\prime} \gamma(1-r)+\gamma^{2}(1-r)(1-p r)\right)}{\left(\beta^{\prime}+\gamma(1-r)\right)(\beta+\gamma(1-p r))} x_{i}
$$

When $p=1$ our results coincide, naturally, with those of M\&S and the optimal strategy is

$$
a_{i}^{*}\left(y, x_{i}\right)=\frac{\beta y+(1-r) \gamma x_{i}}{\beta+(1-r) \gamma}
$$

It is noticeable that from the welfare perspective, given absence of information about $\theta$ the optimal action of an agent is equal to his expectation of $\theta$ given his signals $y$ and $x_{i}$, namely to

$$
a_{i}^{* *}\left(x_{i}, y\right)=\frac{\beta y+\gamma x_{i}}{\beta+\gamma}
$$

Comparing (13) and (14) one can see that because of strategic considerations, in the equilibrium of Morris and Shin (2002), each agent puts inefficiently low weight on his private signal compared to the weight optimal from the welfare 
perspective. Whenever there is higher-order strategic uncertainty, i.e. uncertainty about beliefs about the prior distribution, as we have introduced it, the weight assigned to private information is also inefficiently low compared to (14), this is a robust result, it will be always too low, no matter what $p$ and $\beta^{\prime}$ are, as long as $r \neq 0$. However, if $\beta^{\prime}<\beta$ the weight assigned to the private information may yet be higher as compared to the case without any uncertainty about the prior distribution (like it is in $M \& S$ ), i.e. when $p=1$. Namely,

Proposition 1. In environments with common $p$-belief about the prior distribution where agents have doubts about others' interpretation of public signals, if $\beta^{\prime}<\beta\left(\beta^{\prime}>\beta\right)$ the weight each agent assigns to own private signal is higher (lower) for any $p \in(0,1)$ as compared to the case with $p=1$. Moreover, for $p \in(0,1)$ if $\beta^{\prime}<\beta$ then the weight assigned to private signal is decreasing in $p$ (and it is increasing for $\beta<\beta^{\prime}$ ).

Proof. First part follows from the direct comparison of $\frac{\left.\beta^{\prime} \gamma(1-r)+\gamma^{2}(1-r)(1-p r)\right)}{\left(\beta^{\prime}+\gamma(1-r)\right)(\beta+\gamma(1-p r))}$ with $\frac{(1-r) \gamma}{\beta+(1-r) \gamma}$, the second from differentiation of $\frac{\left.\beta^{\prime} \gamma(1-r)+\gamma^{2}(1-r)(1-p r)\right)}{\left(\beta^{\prime}+\gamma(1-r)\right)(\beta+\gamma(1-p r))}$ with respect to $p$.

This proposition is our first finding - if each agent believes that others are assigning too high weight $\left(\beta<\beta^{\prime}\right)$ to the public signal, he forgoes even further his own private signal and starts to overweigh the value of the public signal. In choosing his action each agent would like to match the best actions of the others (proportionally to the weight $r$ ) and to this end he also starts to act following his suspicion about others' interpretation of all available information.

\section{Uncertainty about $\gamma$}

In this section we consider by contrast the case where there is no common knowledge of how private signals are to be interpreted (and agents commonly agree on the precision of public signal $\beta$ ). For example agents can suspect others to be too overconfident about the quality of their privately observed signals and take those as highly precise reflections of reality. Or by contrast, each agent may believe that others do not have enough of confidence in own signals.

Under absence of common knowledge of $\gamma$ each agent beliefs are

1. Every agent believes with probability one that the precision of private signals is $\gamma$ 
2. Every agent believes that [1] holds with probability $p$ and that with probability $(1-p)$ everyone believes that with probability 1 the precision of the private signals is $\gamma^{\prime}$.

3. Every agent believes that [2] holds with probability $p$ and that with probability $(1-p)$ everyone believes that with probability 1 everyone believes that with probability 1 the precision is $\gamma^{\prime}$.

4. ......

Proceeding as in the previous subsection we obtain the equilibrium strategy as follows 8

$$
a_{i}^{*}\left(y, x_{i}\right)=\frac{\beta \gamma r(1-p)+\beta\left(\beta+\gamma^{\prime}(1-r)\right)}{\left(\beta+\gamma^{\prime}(1-r)\right)(\beta+\gamma(1-p r))} y+\frac{\left.\beta \gamma(1-r)+\gamma \gamma^{\prime}(1-r)(1-p r)\right)}{\left(\beta+\gamma^{\prime}(1-r)\right)(\beta+\gamma(1-p r))} x_{i}
$$

As before, the weight that each agent assigns to his own private signal is inefficiently low compared to what he should assign given just the informational constraints. But if each agent believes that others are likely to value their private signals more than those deserve (i.e. that $\gamma^{\prime}<\gamma$ ) each agent assigns a higher weight to his own private signal as compared to the case with $p=1$.

Proposition 2. In environments with common p-belief about the prior distribution, where agents have doubts about others' interpretation of private signals, if $\gamma<\gamma^{\prime}$ $\left(\gamma>\gamma^{\prime}\right)$ the weight each agent assigns to own private signal is higher (lower) under any $p \in(0,1)$ as compared to the weight assigned under $p=1$. Moreover, for $p \in(0,1)$ the weight assigned to the private signal is increasing in $p$ if $\gamma>\gamma^{\prime}$ and decreasing otherwise.

Proof. Similar to the proof of the previous proposition.

\footnotetext{
${ }^{8}$ For this it is enough to unfold some first orders of iterated expectations, then one can see that in 97 only $q$ and $C$ change to become $q^{\prime}:=\frac{\gamma^{\prime}}{\alpha+\beta+\gamma^{\prime}}$ and $C^{\prime}:=\frac{\alpha \mu+\beta y}{\alpha+\beta+\gamma^{\prime}}$. Then, substituting those expression in (10) together with $k, B, A$ unchanged (and $\alpha=0$ ) one obtains the equilibrium (15).
} 


\subsection{Optimal Precision of Public Information}

We study next what is the optimal degree of transparency given the equilibrium strategies and given agents' uncertainty about others' interpretation of public and private signals. First we consider the case where there is no common knowledge of the precision of the public signal.

Uncertainty about Interpretation of the Public Signal Given the equilibrium action in (12) the social welfare function is

$$
E\left[S W^{p}(.)\right]=-\frac{\left(\beta^{\prime} \gamma(1-p)+\beta\left(\beta^{\prime}+\gamma(1-r)\right)\right)^{2}+\gamma(1-r)^{2} \beta\left(\beta^{\prime}+\gamma(1-p r)\right)^{2}}{\beta\left(\beta^{\prime}+\gamma(1-r)\right)^{2}(\beta+\gamma(1-p r))^{2}}
$$

Our first preliminary result is quite general and holds for arbitrary $\beta^{\prime}$ and $\beta$, social welfare improves from presence of higher-order uncertainty, i.e. if $p$ goes down from 1, only if $\beta^{\prime}<\beta$ (other things being equal):

Proposition 3. The increase of dispersion in higher-order uncertainty, as measured by decrease in $p$, increases expected social welfare if and only if $\beta^{\prime}<\beta$.

Proof. Follows from differentiation of 16 with respect to $p$.

We did not specify much until now where the parameter $\beta^{\prime}$ comes from. In principle $\beta^{\prime}$ can be related to $\beta$ by some function $\beta^{\prime}=f(\beta)$. The function $\beta^{\prime}=$ $f(\beta)$ can be an exogenous property of the environment, i.e. just agents' belief types, how a given public signal is translated into a common $p$-belief. It is realistic, furthermore, that if the precision of public signal increases, agents would believe that others reacting to this "in a right direction" and take the public signal as a somewhat more precise. Hence $f(\beta)$ is an increasing function of $\beta$. In what follows, we will work with a linear function and define $\beta^{\prime}=f(\beta)=s \beta$ or

$$
s:=\frac{\beta^{\prime}}{\beta}
$$

For the moment, the parameter $s$ is treated as fixed and exogenous, later we consider it to be a part of the optimal strategy how the public signal should be disseminated.

With $\beta^{\prime}=s \beta$, the equilibrium strategy (12) can be rewritten as 
$a_{i}^{*}\left(y, x_{i}\right)=\frac{\left.\beta s \gamma r(1-p)+\beta^{2} s+\beta \gamma(1-r)\right)}{(\beta s+\gamma(1-r))(\beta+\gamma(1-p r))} y+\frac{\left.\beta s \gamma(1-r)+\gamma^{2}(1-r)(1-p r)\right)}{(\beta s+\gamma(1-r))(\beta+\gamma(1-p r))} x_{i}$

Given this expression and the welfare measure in (2) we can calculate expected welfare at $\theta$ :

$$
E\left[S W^{p}(.)\right]=-\frac{\beta(\beta s+\gamma(1-r+r s(1-p)))^{2}+\gamma(1-r)^{2}(\beta s+\gamma(1-p r))^{2}}{(\beta s+\gamma(1-r))^{2}(\beta+\gamma(1-p r))^{2}}
$$

First, we disentangle the case where the presence of any imperfectly precise public signal (i.e. $\beta<\infty$ ) reduces welfare as compared to no signal at all:

Proposition 4. The case $\beta=\infty$ notwithstanding, the optimal precision of the public signal $\beta$ is equal to zero when the following holds:

- $\beta \leq \gamma, \bar{r}(\beta, \gamma, p)<r<1, s>\bar{s}(\beta, \gamma, r, p), 0<p<\frac{\beta+\gamma}{2 \gamma}$

- $\beta \leq \gamma, \bar{r}(\beta, \gamma, p)<r<\frac{\beta+\gamma}{2 \gamma p}, s>\bar{s}(\beta, \gamma, r, p), \frac{\beta+\gamma}{2 \gamma}<p<1$

- $\beta \leq \gamma, \frac{\beta+\gamma}{2 \gamma p}<r<1, s>0, \frac{\beta+\gamma}{2 \gamma}<p<1$

Proof. Straightforward from comparing (17) evaluated at $\beta=0$ with (17) for arbitrary $\beta<\infty$. The details of thresholds $\bar{s}(\beta, \gamma, r, p)$ and $\bar{r}(\beta, \gamma, p)$ are given in the Appendix.

This proposition is intuitive, it is better to provide no public signal at all if its precision cannot be above the precision of private signals. Second, coordination motives should be important ( $r$ is relatively high), as first and second bullet points indicate it. Furthermore, there should be pronounced overreaction to the public signal (as measured by high $s$ and low $p$, although in the last two bullet points high $r$ suffices to reduce value of communication regardless of $s$ and $p$ ).

Proposition 5. (i) Expected social welfare is always increasing in $\gamma$.

(ii) Expected social welfare is decreasing in $\beta$ for $\beta \in[0, \bar{\beta}), r \in(\bar{r}, 1], s \in(\bar{s}, \infty)$ and $p \in[0,1]$, with $\bar{\beta} \leq \gamma$.

Proof. Details are spelled out in the Appendix. 


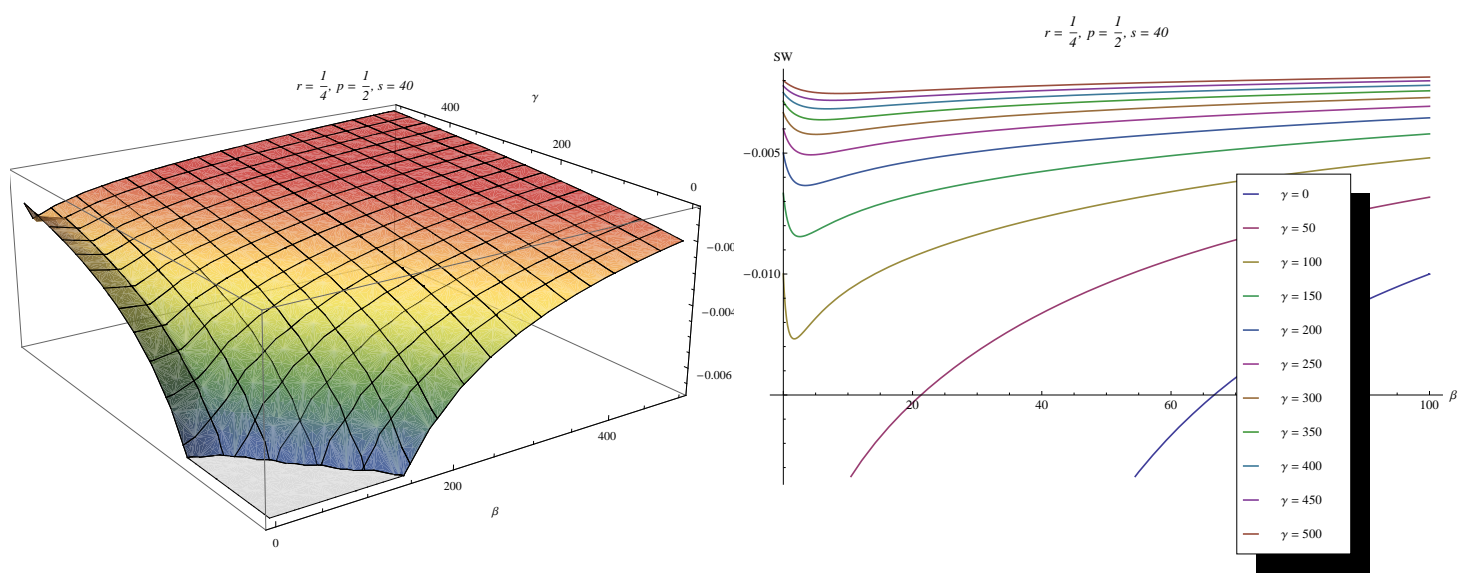

Figure 1: Corollary example: Social welfare is decreasing in $\beta$ for $r=\frac{1}{4}$

This means that whenever agents expect with a sufficiently high probability (i.e. $(1-p)$ is high) that others take the public signal too literally, i.e. as too informative $(s>1)$, social welfare is decreasing in precision of public information over a larger set of parameters, and more specifically even for $\beta$ relatively high compared to $\gamma$ (thus the critique of Svensson (2006) fails in our environment)

Recall that the main policy implication of Morris and Shin (2002) was that if private signals are sufficiently precise and if the central bank cannot improve the precision beyond a threshold, it can be optimal to set $\beta=0$, i.e. no information should be released. In our case, the above results suggest that if $s$ is sufficiently large $(s>1)$ and agents' doubts, as measured by $(1-p)$ are large (i.e. $p$ low), even for $r$ relatively low it may be optimal to set $\beta=0$. Namely, in Morris and Shin (2002) social welfare is always increasing in $\beta$ for $r<\frac{1}{2}$. The higher-order uncertainty unsettles this result:

Corollary 6. There exists $\bar{s}>>1$ and $\bar{p}$ such that if $s>\bar{s}$ and $p<\bar{p}$ even for $r<\frac{1}{2}$ social welfare is decreasing in $\beta$.

The Corollary is illustrated in Figure 1.

An intuition behind this result is relatively simple: $s>1$ is a multiplier of a negative effect that the public signal may have, if $\beta$ is relatively low and its marginal increase is welfare decreasing, $s$ worsens the situation further: not only agents put too much weight on a very noisy public signal but they also believe that others put too high weight on it and it provides them with further incentives to put an even higher weight on the (very likely wrong) public signal. The higher 
is $s$ the smaller is $r$ which introduces the negative effect of communication for low $\beta$.

Influencing Higher Order Beliefs about the Public Signal Up to now we have treated the function $\beta^{\prime}=f(\beta)=s \beta$ as exogenous. However, arguably, a given public signal may have a multidimensional impact and influence both $\beta$ and, to a certain extent, higher-order beliefs and commonality of understanding, i.e. s: in the case of communication by a central bank, it can explain its information in a way which not only improves on agents' understanding of the central bank's signal, but which also influences their perception of how others' might have understood the signal. For example, providing some very technical information increases precision of the public signal for a sophisticated agent, but at the same time it may also make each agent think that others are not as good at deciphering too complicated message and hence they are likely to disregard the information contained in the public signal. The following proposition says that it is always optimal for the central bank, if feasible, to set $s=0$

Proposition 7. For any finite $\beta$, any $r \in(0,1), p \in(0,1), \gamma>0$, social welfare is decreasing in s, i.e. for any finite $\beta$ it is optimal to set $s=0$ or any feasible $s_{\text {min }}$.

Proof. Follows again from taking a derivative of $E\left[S W^{p}().\right]$ with respect to $s$ and solving a system of inequalities.

Thus, notwithstanding the case where $\beta=\infty$, i.e. the public signal is fully revealing about $\theta$, it is always optimal for the central bank to muddle the perceived precision of the information it reveals. Putting differently, if $\beta$ cannot be infinite, there is always welfare improving obfuscation of information provided it affects agents' second (and higher) order beliefs by making them think that others think the public information is totally valueless.

Note however that setting $s=0$ does not necessarily imply that social welfare is will be always increasing in $\beta$ :

Proposition 8. Social welfare is decreasing in $\beta$ even for $s=0$ provided $\frac{\gamma}{\beta}>\frac{1}{(1-p r)(2 p r-1)}$, $\frac{1}{2 r}<p<1, \frac{1}{2}<r<1$.

The reason why setting $s=0$ does not always solve fully the problem of overreaction to the public signal is that for $p$ close to 1 differences in $s$ have little influence on the equilibrium strategy and approximately the same comparative statics as in Morris and Shin (2002) holds. 
Uncertainty about Interpretation of Private Signals Next we provide the welfare analysis for the case where agents are uncertain how other agents are interpreting their private signals. The situation turns out to be diametrically opposite to the case where agents are uncertain how others are interpreting public signals. Again we can define

$$
t:=\frac{\gamma^{\prime}}{\gamma}
$$

In this case expected social welfare is equal to

$$
E\left[S W^{p}(.)\right]=-\frac{\beta(\beta+\gamma(t+r(1-p-t)))^{2}+\gamma(1-r)^{2}(\beta+\gamma t(1-p r))^{2}}{(\beta+t \gamma(1-r))^{2}(\beta+\gamma(1-p r))^{2}}
$$

Revelation of information publicly may be again welfare decreasing for any $\beta<\infty$ :

Proposition 9. The case $\beta=\infty$ notwithstanding, the optimal precision of the public signal $\beta$ is equal to zero when the following holds:

- $\beta \leq \gamma, \bar{r}(\beta, \gamma, p)<r<1,0<t<\overline{\bar{t}}(\beta, \gamma, r, p), 0<p<\frac{\beta+\gamma}{2 \gamma}$

- $\beta \leq \gamma, \bar{r}(\beta, \gamma, p)<r<\frac{\gamma+\beta}{2 \gamma p}, 0<t<\overline{\bar{t}}(\beta, \gamma, r, p), \frac{\beta+\gamma}{2 \gamma}<p<1$

- $\beta \leq \gamma, \frac{\gamma+\beta}{2 \gamma p}<r<1, t>0, \frac{\beta+\gamma}{2 \gamma}<p<1$

Proof. Straightforward from comparing (18) evaluated at $\beta=0$ and for any $\beta>0$. The exact expressions for $\bar{r}$ and $\overline{\bar{t}}$ are given in the Appendix.

At first sight this proposition might look surprising as the optimality of providing any public signal at all depends on the value of $t$ (if $\beta$ is relatively low, when $r$ and $p$ are high, $t$ is irrelevant). However this is to be expected given that the value of a positive precision of the public signal depends on relative weights agents put on private and on public signals when choosing their optimal actions. The weight assigned to the public signal, no matter how big or how small, will reflect agent $i$ thinking about others valuation of the public signal which itself depends on $i$ 's consideration of the precision of $(-i)^{\prime} s$ private signals as perceived by $(-i)^{\prime} s$ and thus it will depend on $t$.

The next proposition provides a general result when welfare is decreasing in $\beta:$ 
Proposition 10. (i) The value of $E\left[S W^{p}().\right]$ is always increasing in $\gamma$ and $t$.

(ii) For $0 \leq t<1(t>1)$ the value of $E\left[S W^{p}().\right]$ is increasing (decreasing) in $p$, i.e. increase in uncertainty about others' interpretation of private signals, as measured by decrease in $p$, is welfare beneficial only if $t>1$.

(iii) Expected social welfare is decreasing in $\beta$ for $\beta \in[0, \bar{\beta}), r \in(\bar{r}, 1), t \in[0, \bar{t})$ and $p \in(0,1)$.

Proof. Details are in the Appendix.

Hence, the more agents believe that others take their private signals as too informative, the smaller is the effect of overreaction to public signal. Put differently, the overreaction to public information is dampened by agents' believing that others are too concentrated on their private signals. However opposite is true if everyone believes that others disbelieve their private signals too much, they disregard those signals as a result and and rely exclusively on publicly available signals. An imperfectly informative public signal thus can aggravate the situation, as on the one hand it makes agents to forgo even further their private signals but has too low value on its own.

Similarly to the previous section, it can be shown that even for $r<1 / 2$ social welfare can be decreasing in $\beta$.

Corollary 11. There exists $\bar{\beta}, \bar{t}$ and $\bar{p}$ such that if $t<\bar{t}$ and $p<\bar{p}$, social welfare is decreasing in $\beta$ for $\beta \in[0, \bar{\beta})$ even if $r<\frac{1}{2}$.

This Corollary is illustrated in the Figure 2 .

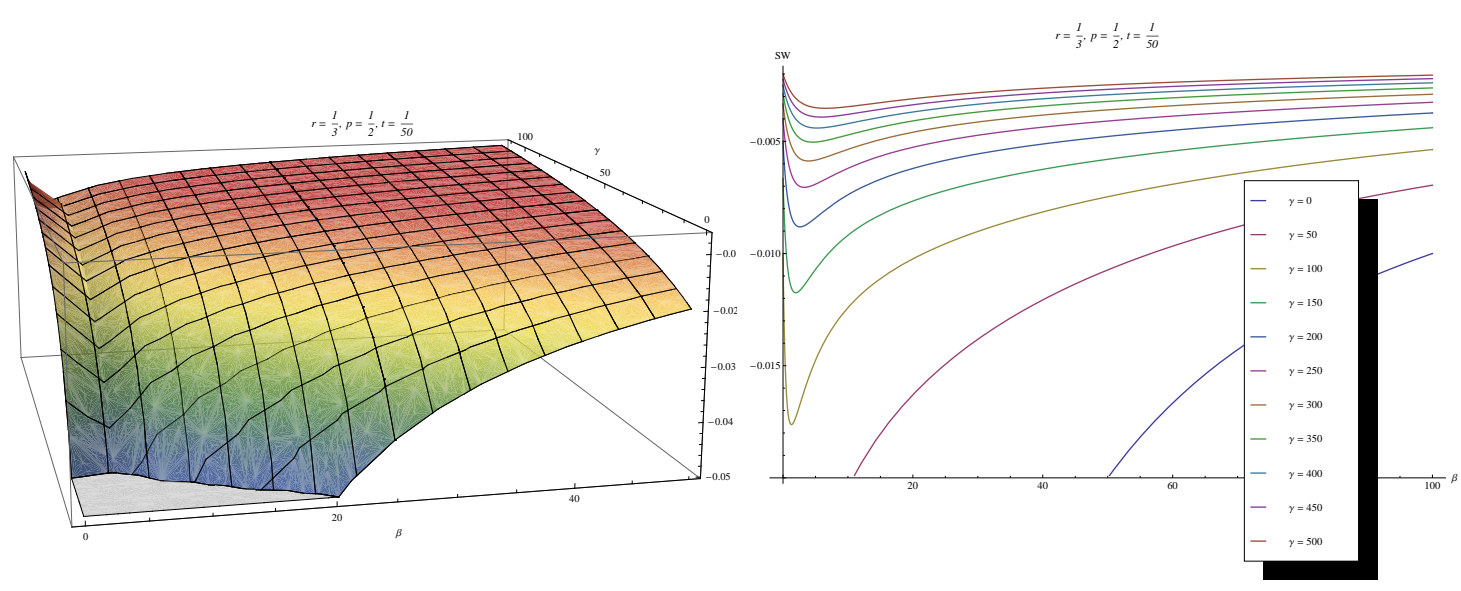

Figure 2: Social welfare with $r=\frac{1}{3}, p=\frac{1}{2}, t=\frac{1}{50}$ 
Thus even when $r$ is low, i.e. coordination with the others is not that important for agents and furthermore agents have doubts only about others' interpretation of private signals, too imprecise public signals may be yet welfare detrimental as compared to no communication at all.

Uncertainty About the Average Error As it has been discussed in the introduction, empirical findings suggest that agents can bear doubts about how others evaluate the expected error of the public signal. Or, similarly, how others evaluate the expected average of private signals' errors. In other words, each agent have the following hierarchy of common $p$-beliefs:

1. Every agent believes with probability one that the joint distribution of $(\theta, y, x)$ is given by $\mathcal{N}(\mu, \Sigma(\alpha, \beta, \gamma))$

2. Every agent believes that [1] holds with probability $p$ and that with probability $(1-p)$ everyone believes that with probability 1 the joint distribution of $(\theta, y, x)$ is given by $\mathcal{N}\left(\mu^{\prime}, \Sigma\right)$, with $\mu^{\prime} \neq \mu$

3. Every agent believes that [2] holds with probability $p$ and that with probability $(1-p)$ that everyone believes that with probability 1 that everyone believes that with probability 1 the joint distribution of $(\theta, y, x)$ is given by $\mathcal{N}\left(\mu^{\prime}, \Sigma\right)$.

4. ......

It turns out that greater transparency is more likely to be welfare increasing:

Proposition 12. Let $\boldsymbol{\mu}^{\prime} \neq \boldsymbol{\mu}$, then an increase in $\beta$ always increases social welfare whenever $p$ is sufficiently low.

Details of the proof are left to the reader.

The intuition behind this proposition is quite simple - the doubts about others beliefs about the average of mistakes in the economy leads to higher discrepancy between the average action and the value of the fundamental, which reduces welfare. Yet, this discrepancy affects agents utility and social welfare only proportionally to $\frac{1}{\beta}$ and as a consequence increase in $\beta$ reduces it. A relatively low $p$ makes the importance of the discrepancy higher in the social welfare, that is why when $p$ is low, even if $\beta$ itself is rather low but $\gamma$ is high (i.e. we are in the region where under $p=1$ increase of $\beta$ would be welfare detrimental), the gains 
to welfare from the reduction in perceived disagreement about the expected error terms are higher compared to losses due to overreaction to a noisy public signal.

The summary of the results in this section suggests that in order to evaluate the entire impact of a more transparent communication one should take into account not only agents' own understanding of public signals but also their perception of others' understanding of private and public signals and also whether others perceive signals as biased. Fine details of those perceptions matter for the optimal policy prescription.

Empirical findings on individual perceptions of their relative abilities suggest that in general agents consider themselves as better decision-makers compared to others. However, this relative confidence in own abilities does not provide much of a hint to answer the question whether "being better" means that others take private or public signals too literally and overweigh them, or whether they do not see those signals and underweigh them. As we have shown these two possibilities and whether such perceptions hold for private or public signals provide very different prescriptions for the optimal communication policy. Furthermore, in a world with the ex ante disagreement, as in the last part of this section, public communication is very important to align ex ante heterogeneous expectations. Sometimes this role of public communication can be much more important than its sins at inducing agents to overreact to a too noisy public signal.

\section{Extensions}

\subsection{Other Welfare Measures}

Woodford (2005) has suggested that the welfare measure in Morris and Shin (2002) is very special, as it accounts only for a discrepancy between agents actions and the fundamental, and instead the following measure is more appropriate:

$$
E\left[S W^{W}(.)\right]=-\frac{1}{(1-r)} E\left[L_{i}\right]
$$

where $L_{i}$ is given by

$$
L_{i}=(1-r)\left(\theta-a_{i}\right)^{2}+r E_{j}\left(a_{i}-a_{j}\right)^{2}
$$


i.e. the welfare measure should take into consideration also the fact that agents fail to coordinate with each other. For example in the game of coordination on investment into a specific industry, when individual agents' return from this investment depend not only on the fundamental value of projects but also on the amount of aggregate investment, it is desirable from the aggregate perspective that many agents invest in the same industry and hence the decision on the optimal degree of precision of a public signal should take into account the potential mismatch in agents actions.

In this case social welfare for the model of Morris and Shin (2002) is equal to

$$
E\left[S W^{W}(.)\right]=-\frac{\beta+\gamma\left(1-r^{2}\right)}{(\beta+\gamma(1-r))^{2}}
$$

and it is always an increasing function of $\beta$ and $\gamma$, no matter what $\gamma$ is, i.e. more communication is always welfare improving.

It turns out that in our setting the results of Woodford do not hold any longer. Substituting equilibrium actions in 19 with $\beta^{\prime}=\beta s$ we obtain the following expression

$$
\begin{gathered}
E\left[S W^{W}(.)\right]=-\frac{\left(\gamma^{2}(1-r)(1-p r)+\beta \gamma s(1-s)\right)^{2}}{\gamma(\beta+\gamma-\gamma p r)^{2}(\gamma-\gamma r+\beta s)^{2}}- \\
-\frac{2 r\left(\gamma^{2}(1-r)(1-p r)+\beta \gamma s-\beta \gamma r s\right)^{2}}{\gamma(1-r)(\beta+\gamma(1-p r))^{2}(\gamma-\gamma r+\beta s)^{2}}-\frac{\left(\beta \gamma(1-r)+\beta^{2} s+\beta \gamma r s(1-s)\right)^{2}}{\beta(\beta+\gamma-\gamma p r)^{2}(\gamma-\gamma r+\beta s)^{2}}
\end{gathered}
$$

Welfare maybe yet decreasing in $\beta$, even for $r<1 / 2$, as Figure 3 demonstrates. Furthermore welfare maybe decreasing also in $\gamma$ - the effect which was never available in the previous section, no matter how big or small were parameters $(p, r, s, t)$.

The reason for this result is the following. The new welfare measure accounts for two effects of increased precision of public information: one it is the part of Morris and Shin (2002) where for $\beta$ low welfare can be decreasing in $\beta$, another is the always positive gain in welfare due to increased capacity to coordinate with each other. With $p=1$, the second effect of increased $\beta$ has been always larger than the first. Under common $p$-belief about $\beta$ with $p<1$ the first effect can be still dominating, if $\beta^{\prime}=\beta s$ is sufficiently high due to high $s$.

The result changes compared to the previous Section, where it was always optimal to set $s=0$, for any feasible $\beta$. For example, provided $\beta>\gamma$ is feasible, 


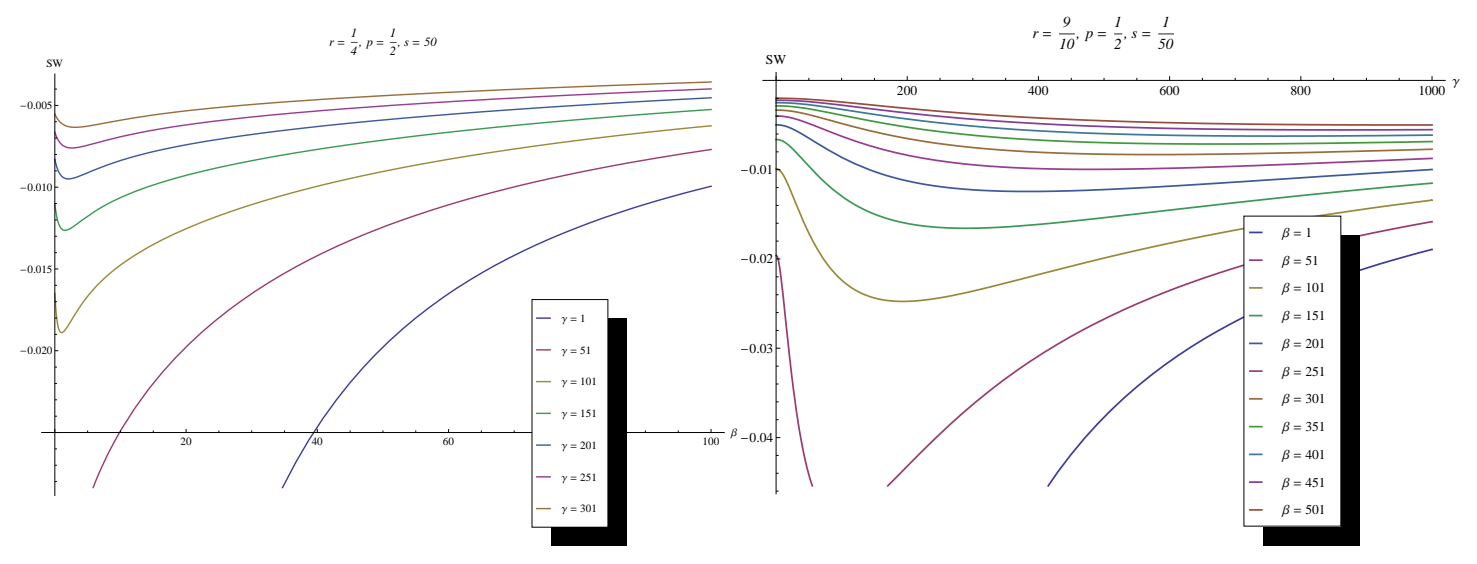

Figure 3: Social welfare under uncertainty about $\beta$ can be: (i) decreasing in $\beta$; (ii) decreasing in $\gamma$.

the value of $s$ should grow to the maximum feasible level. That is, it is valuable to induce the strong belief in a very high precision of the public signal (for $p$ fixed).

Proposition 13. Expected social welfare $E\left[S W^{W}().\right]$ is increasing in s if the following holds:

- $\beta<\gamma, s \in\left[0, \frac{2 \gamma}{\gamma-\beta}\right], p \in(0,1), r \in(0,1)$

- $\beta<\gamma, s \in\left(\frac{2 \gamma}{\gamma-\beta}, \infty\right), p \in\left(\frac{2 \gamma+s(\beta-\gamma)}{\gamma(1+r-s)}, 1\right), r \in(0,1)$

- $\beta>\gamma, s \in(0,1), p \in(0,1), r \in(0,1)$

Furthermore, if welfare is measured according to (19) there is a discontinuity at $s=0$, namely, $E\left[S W^{W}().\right]$ is decreasing in $\gamma$ for $\gamma \in\left(0, \frac{\beta(3 r-1-p r(1+r))}{(1-p r)(1+r)}\right), r \in\left(0, \frac{1}{3}\right)$, $p \in\left(0, \frac{3 r-1}{r(r+1)}\right)$ and any $\beta>0$. On the other hand, at $s=0$ social welfare is always increasing in $\beta$. Thus, under this different measure, if $\gamma$ is relatively low, setting $s=0$ wipes out the value of private signals from the welfare perspective (if those are of a low precision).

When there is uncertainty how others are interpreting their private signals, social welfare can be decreasing in $\beta$ and also decreasing in $\gamma$. Again, aggregating individual strategies according to (19) and substituting for $\gamma^{\prime}=t \gamma$ we obtain the following expression for expected social welfare

$$
E\left[S W^{W}(.)\right]=-\frac{\left(\beta^{2}-\beta \gamma(-1+p) r-\beta \gamma(-1+r) t\right)^{2}}{\beta(\beta+\gamma(1-p r))^{2}(\beta+\gamma s-\gamma r t)^{2}}-
$$


$-\frac{\left(\beta \gamma-\beta \gamma r+\gamma^{2}(1-r)(1-p r) t\right)^{2}}{\gamma(\beta+\gamma(1-p r))^{2}(\beta+\gamma t(1-r))^{2}}-\frac{2 r\left(\beta \gamma-\beta \gamma r+g^{2}(1-r)(1-p r) t\right)^{2}}{\gamma(1-r)(\beta+\gamma(1-p r))^{2}(\beta+\gamma t(1-r))^{2}}$

Figure 4 demonstrates the case where welfare is decreasing in $\gamma$ under uncertainty about $\gamma$.
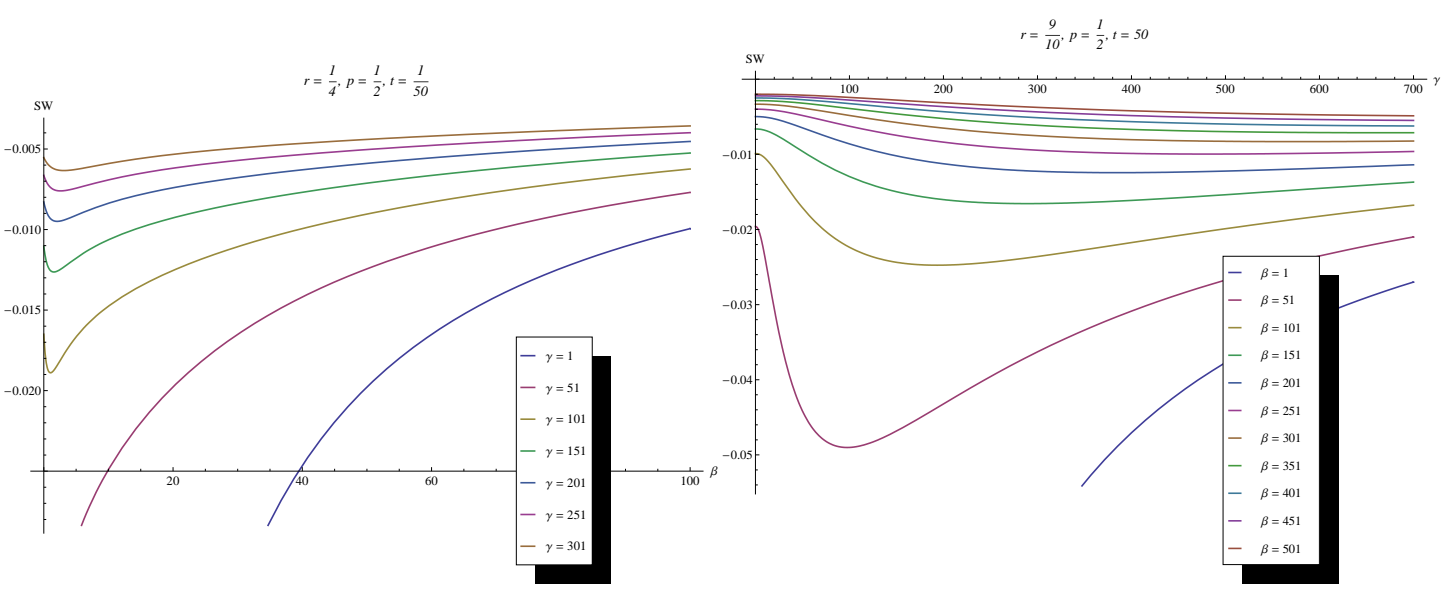

Figure 4: Social welfare under uncertainty about $\gamma^{\prime}$ : (i) can be decreasing in $\beta$; (ii) can be decreasing in $\gamma$.

This result originates from miscoordination among agents and the non-zero weight that the welfare measure puts on it. And if agents believe that others overreact to individual signals, but those signals themselves are quite noisy $(\gamma$ is very low), each agent takes into account own private signals unjustifiably too much, which results in welfare losses.

To summarize, under common $p$-beliefs about the data generating process, the alternative welfare measure as in Woodford (2005) results in different predictions compared to the case where the prior is commonly known. Contrary to the results in Woodford (2005), doubts about others abilities to interpret all information properly can add to welfare losses through the channel of the unjustifiably high weight given to private signals withholding agents from efficient coordination with each other through the public signal. This effect never appears when one considers the welfare measure as in Morris and Shin (2002) regardless if $p=1$ or $p<1$. 


\subsection{Other forms of higher order uncertainty}

We have considered the case where the prior which is, put roughly, $(1-p)$ believed is the same for each order of doubts (agents put probability 1 on others believing with probability $1 \ldots$ in $\beta^{\prime}$ or $\gamma^{\prime}$ ) and moreover it is the same across agents. The question arises whether the conclusions of the paper would be different had we allowed for a richer framework, i.e. a richer belief structure and higher order doubts about the prior. It seems that while computationally the task of solving for the equilibrium with heterogeneous $\beta_{i}^{\prime}$ becomes even more convoluted, conclusions would not change much. The reason for this is inherent continuity of the equilibrium action in each order of expectations about others' expectations. Moreover the impact that each subsequent order of doubts has on the equilibrium action is exponentially diminishing with each additional level of beliefs. This means that even if some higher-order expectation at a level $k$ does jump drastically, unless this jump outweighs significantly the weight $r^{k}$ (recall that $r<1$ ) assigned to that order of expectation, the total effect of the jump on the equilibrium strategy would not be too high.

Our model is general enough to allow for common knowledge of disagreement in a population of agents divided into two groups, where each agent considers that there is a fraction $k$ of agents who believe that the precision of public signal is some $\beta^{\prime \prime}$ and another fraction $(1-k)$ who believe the precision to be $\beta^{\prime \prime \prime}$. Then, it is enough to substitute for $\beta^{\prime}=k \beta^{\prime \prime}+(1-k) \beta^{\prime \prime \prime}$ and let $p=0$ in the above analysis to obtain the desired result. In this case results depend on how an increase in $\beta$ influences $\beta^{\prime \prime}$ and $\beta^{\prime \prime \prime}$. The main trade-off is that again an increase in the precision of public information can be welfare detrimental due to too much noise and agents over-reaction to it. Yet, if both functions $\beta^{\prime \prime}(\beta)$ and $\beta^{\prime \prime \prime}(\beta)$ are monotonic in $\beta$, an increase in $\beta$ reduces simultaneously the discrepancy between $\beta^{\prime \prime}$ and $\beta^{\prime \prime \prime}$, which itself increases the welfare. The optimal policy, hence, should to be weighting off those two effects.

\section{Conclusions}

In this paper we characterize equilibrium behaviour and social welfare for a beauty-contest game in a class of environments where agents' beliefs fail to be consistent with common knowledge of the data-generating process. Depending on the relevant welfare measure, there may be a scope for public communication 
to play on this absence of common knowledge of the data-generating process. Specifically if the welfare objective is to make agents align their actions with the fundamental (and not with each other), then the central authority should do its best to increase the precision of the public signal given its correct interpretation by each agent $i$ and it should do its best to make each agent $i$ believe that others did not understand much about the actual meaning of the public signal. By contrast when the welfare measure accounts for both, matching the fundamental and alignment of agents' actions with each other, then, depending on values of the parameters, public communication might need to be as clear as possible in order to lead to the best feasible common understanding of public signals.

Future research should provide empirical and experimental validation of different structures of higher-order beliefs considered in this section. Also, similarly to the paper by Myatt and Wallace (2011) it would be desirable to investigate under which conditions agents are failing endogenously to have common knowledge of the data-generation process due to costly inference about the actual process (this agenda is currently work in progress). Another possible interesting extension is to allow agents' actions to be strategic substitutes, e.g., by introducing absence of common knowledge of the prior into a more general framework of Angeletos and Pavan (2007).

\section{References}

Angeletos, G.-M., And A. PaVAn (2007): "Efficient Use of Information and Social Value of Information," Econometrica, 75, $1103-1142$.

Batson, C. (1975): "Rational Processing or Rationalization? The Effect of Disconfirming Evidence on a Stated Religious Belief," Journal of Personality and Social Psychology, 32, 176-184.

Ben-David, I., J. Graham, And C. Harvey (2010): “Managerial Miscalibration," Working paper, NBER.

Benoit, J., And J. Dubra (2011): “Apparent Overconfidence," Econometrica, 79, 1591-1625.

Burks, S., J. CARpenter, L. Goette, and A. Rustichini (2013): “Overconfidence and Social-Signaling," Review of Economic Studies, pp. xxx-xxx. 
Cornand, C., and F. Heinemann (2008): “Optimal Degree of Public Information Dissemination," The Economic Journal, 118, 718 - 742.

Glaser, M., And M. Weber (2007): "Overconfidence and Trading Volume," Geneva Risk Insurance Review, 32, 1-36.

HeLLWIG, C. (2005): "Heterogeneous Information and the Welfare Effect of Public Information Disclosure," Working paper, UCLA.

HellWig, C., AND L. VeldKAmP (2009): “Knowing What Others Know: Coordination Motives in Information Acquisition," Review of Economic Studies, 76, 223 -251 .

Kandel, E., And N. Pearson (1995): “Differential Interpretation of Public Signals and Trade in Speculative Markets," Journal of Political Economy, 103, 831 872.

LAHIRI, K., AND X. SHENG (2008): "Evolution of Forecast Disagreement in a Bayesian Learning Model," Journal of Econometrics, 144, 325 - 340.

Lord, C., L. Ross, And M. Lepper (1979): “Biased Assimilation and Attitude Polarization: The effects of Prior Theories on Subsequently Considered Evidence," Journal of Personality and Social Psychology, 37, 2098 - 2109.

Monderer, D., And D. SAmet (1989): “Approximating Common Knowledge with Common Belief," Games and Economic Behaviour, 1, 170 - 190.

MONTIER, J. (2006): “Behaving Badly," Research paper, DrKW.

MORRIS, S. (1995): "The common prior assumption in economic theory," Economics and Philosophy, 11, 227-253.

MORRIS, S., AND H. SHIN (2002): "Social Value of Public Information," American Economic Review, 92, $1521-1534$.

MORRIS, S., AND H. SHIN (2007): “Optimal Communication," Journal of European Economic Association, 5, 594-602.

MyatT, D., And C. WAllace (2009): "On the Sources and Value of Information: Public Announcement and Macroeconomic Performance," Working paper, University of Oxford. 
(2011): "Endogenous Information Acquisition in Coordination Games," Review of Economic Studies, pp. 1 - 38.

Odean, T. (1998): "Volume, Volatility, Price and Profit When all Traders are Above Average," Journal of Finance, 53, 1888 - 1934.

RosA, C., AND G. Verga (2008): “The Impact of Central Bank Announcements on Asset Prices in Real Time," International Journal of Central Banking, 4, 175217.

SVENSON, O. (1981): "Are we all less risky and more skillful than our fellow drivers," Acta Psychologica, 47, 143 - 148.

SVENSSON, L. (2006): "Social Value of Public Information: Morris and Shin (2002) is Actually Pro-Transparency, not Con," American Economic Review, 96, 448 452 .

Woodford, M. (2005): “Central Bank Communication and Policy Effectiveness," Working paper, NBER.

\section{A Appendix}

\section{A.1 Details of Proofs}

Proposition 4: The thresholds are the following:

$$
\bar{r}=\frac{\beta(\beta+\gamma)}{\gamma(\beta+\gamma+p(\beta-\gamma))}, \bar{s}=\frac{\gamma(1-r)(2 \gamma p r-\gamma-\beta)}{\beta(\gamma+\beta)-\gamma r(\beta+\gamma+p(\beta-\gamma))}
$$

\section{Proposition 5 :}

It is notoriously complicated to obtain the closed form analytical solution to the system of inequalities defined by $\frac{\partial}{\partial \beta} E S W<0$ and all the feasibility constraints on values of parameters. However we are yet able to specify some necessary conditions on parameters under which the social welfare is decreasing in $\beta$. For this we observe that the second summand in (17) is monotonically increasing in $\beta$ and the first summand is decreasing in $\beta$ if the following holds:

- $\beta \leq \gamma, \frac{\gamma-\beta}{\gamma}<r<1,0<s<\hat{s}(\beta, \gamma, r), 0<p<\hat{p}(\beta, \gamma, r, s)$

- $\beta \leq \gamma, \frac{(\gamma-\beta) \beta}{\gamma(3 \beta+\gamma)}<r<\frac{\gamma-\beta}{\gamma}, 0<s<\hat{s}(\beta, \gamma, r), 0<p<1$ 
- $\beta \leq \gamma, \frac{(\gamma-\beta) \beta}{\gamma(3 \beta+\gamma)}<r<\frac{\gamma-\beta}{\gamma}, \hat{s}(\beta, \gamma, r)<s, \hat{p}(\beta, \gamma, r, s)<p<1$

with $\hat{s}(\beta, \gamma, r)=\frac{1}{2}\left(\sqrt{\frac{\gamma^{3}(\gamma-\beta)(1-r)^{2} r(8 \beta(\beta+\gamma)-(\gamma-b) r}{\beta^{2}(\beta(\beta-\gamma)+\gamma(3 \beta+\gamma) r)^{2}}}+\frac{(\gamma-\beta) \gamma(1-r)(2 \beta+\gamma r)}{\beta(\beta(\beta-\gamma)+\gamma(3 \beta+\gamma) r)}\right)$;

Thus, the thresholds given in the Proposition 5 coming from the sum of a strictly increasing function and the function having the above critical points would lie within the above thresholds. We illustrate in addition the proposition for different constellations of $(\beta, \gamma, p, s)$ at the second part of the appendix.

Proposition 9 The thresholds are the following:

$$
\bar{r}=\frac{\beta(\beta+\gamma)}{\gamma(\beta+\gamma+p(\beta-\gamma))}, \overline{\bar{t}}=\frac{\beta(\beta+\gamma)-\gamma r(\beta+\gamma+p(\beta-\gamma))}{\gamma(1-r)(2 \gamma p r-\gamma-\beta)}
$$

\section{A.2 The case with uncertainty about both $\beta$ and $\gamma$}

We assume again that $\beta^{\prime}=s \beta$ and $\gamma^{\prime}=t \gamma$, the social welfare in this case is equal to:

$$
E S W=-\frac{\left(\gamma(1-r)^{2}(\beta s+\gamma(1-p r) t)^{2}+\beta(\beta s+\gamma(t+r((1+p) s-t)))^{2}\right)}{(\beta+\gamma(1-p r))^{2}(\beta s-\gamma(1-r) t)^{2}}
$$

as one can see the effect of $s$ and $t$ on the expected social welfare are "additive" (i.e. there is no direct term involving a product of $s$ and $t$ ). Thus, there is no loss in generality by studying effects of $\beta^{\prime}$ and $\gamma^{\prime}$ separately.

\section{A.3 Illustration of Propositions 5 and 10.}

First three pages illustrate Proposition 5 and subsequent three pages illustrate 10 . 

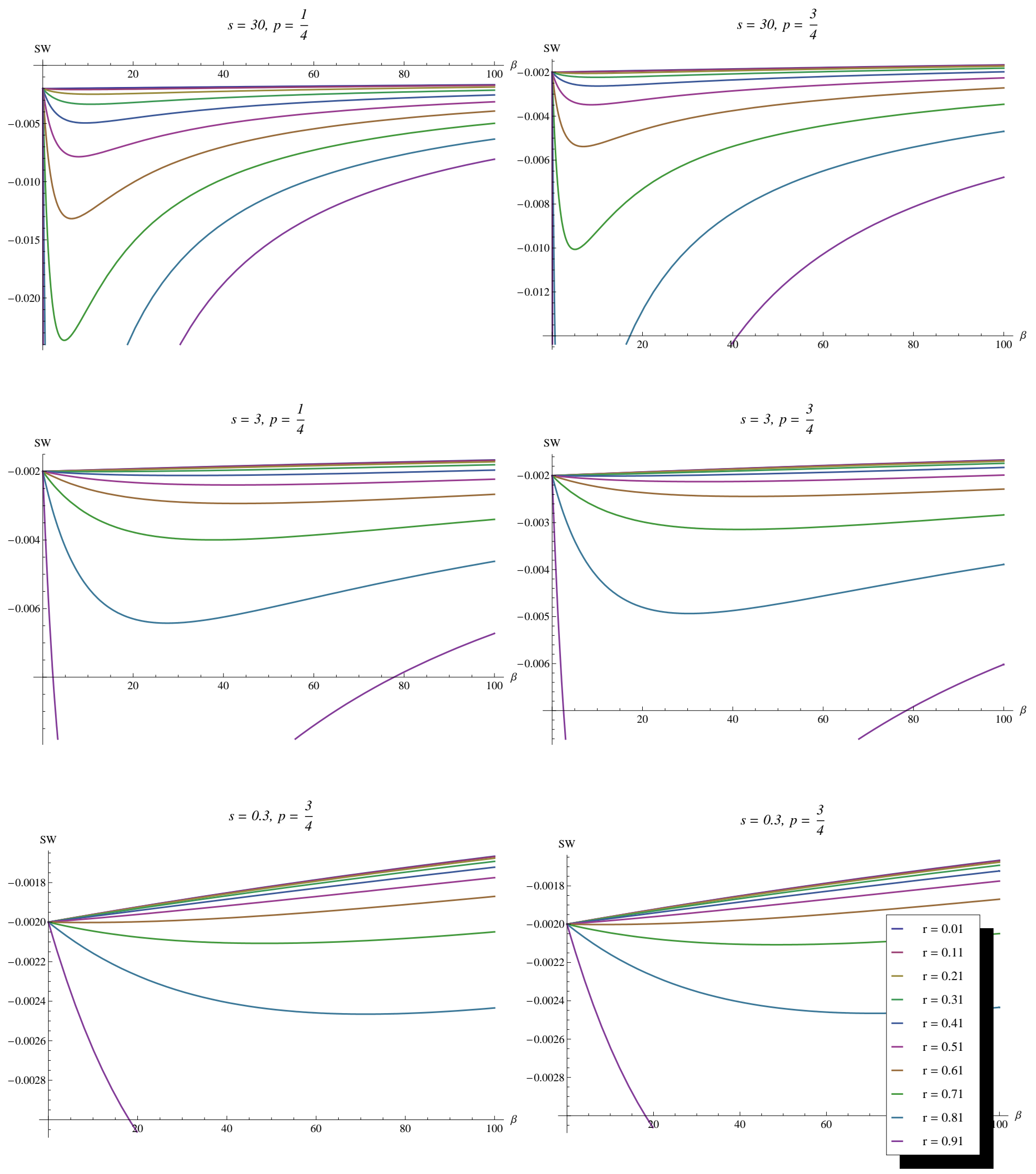

Figure 5: Proposition 5. Varying $r$ 

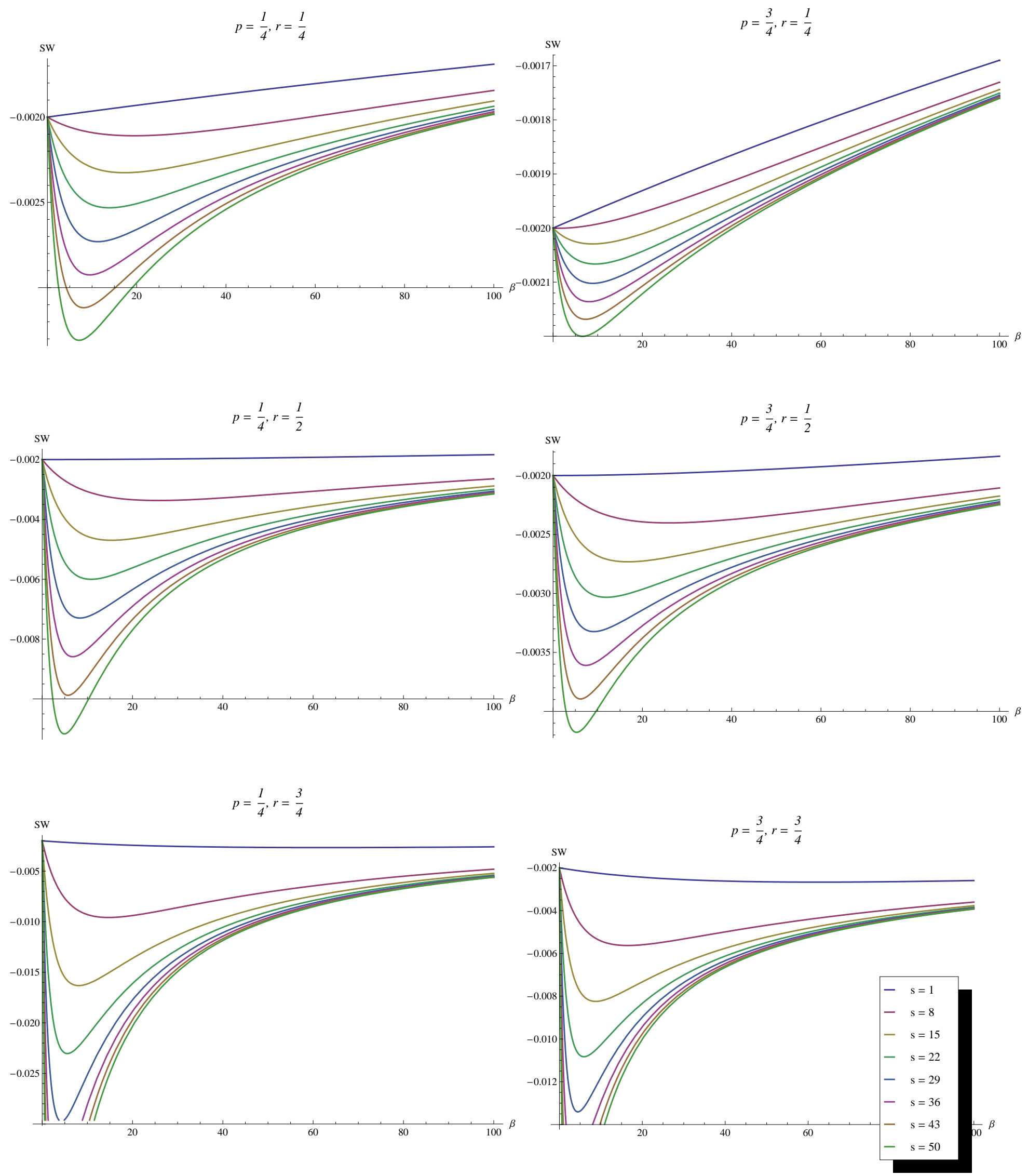

Figure 6: Proposition 5(continued): Varying $s$ 

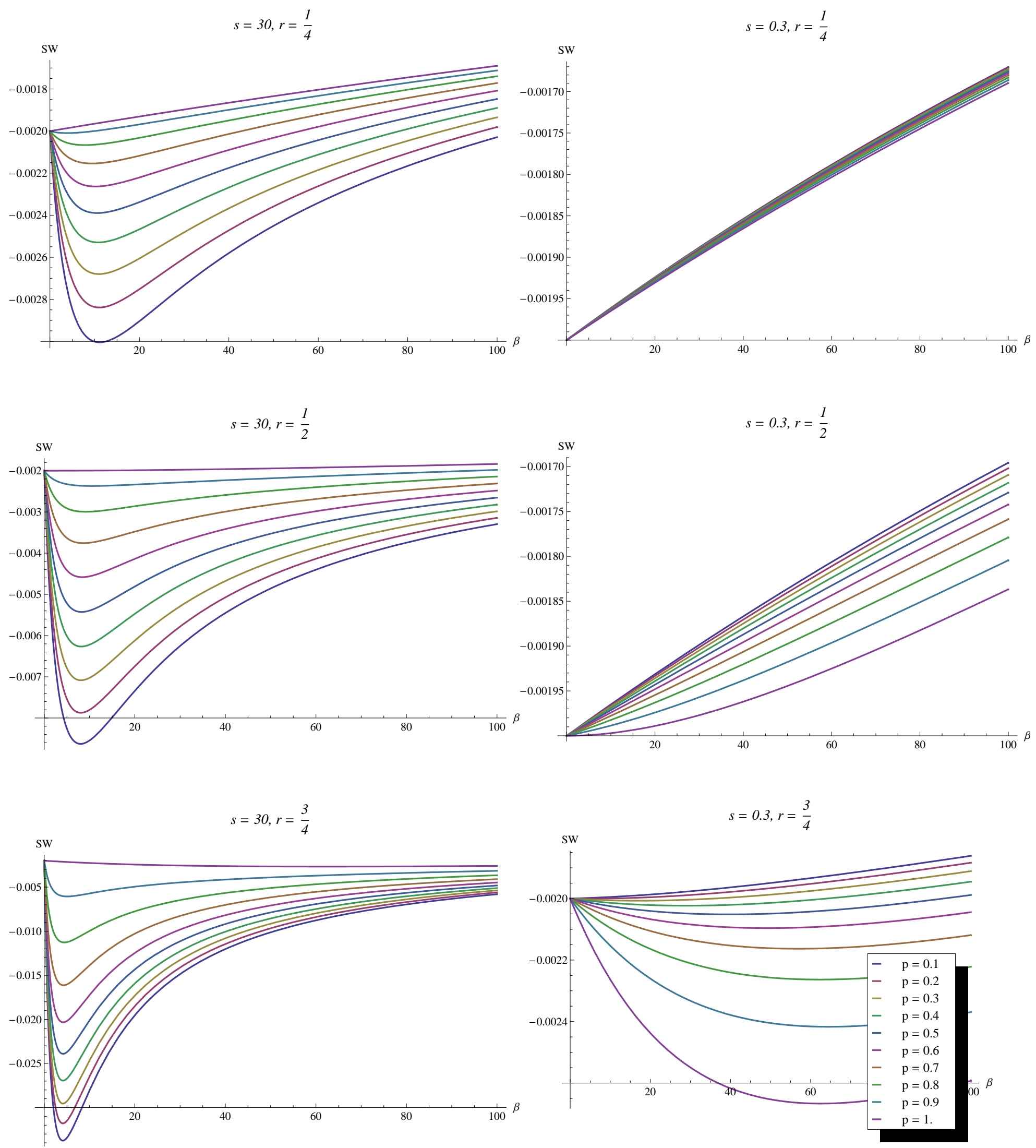

Figure 7: Proposition 5(continued): Varying $p$ 

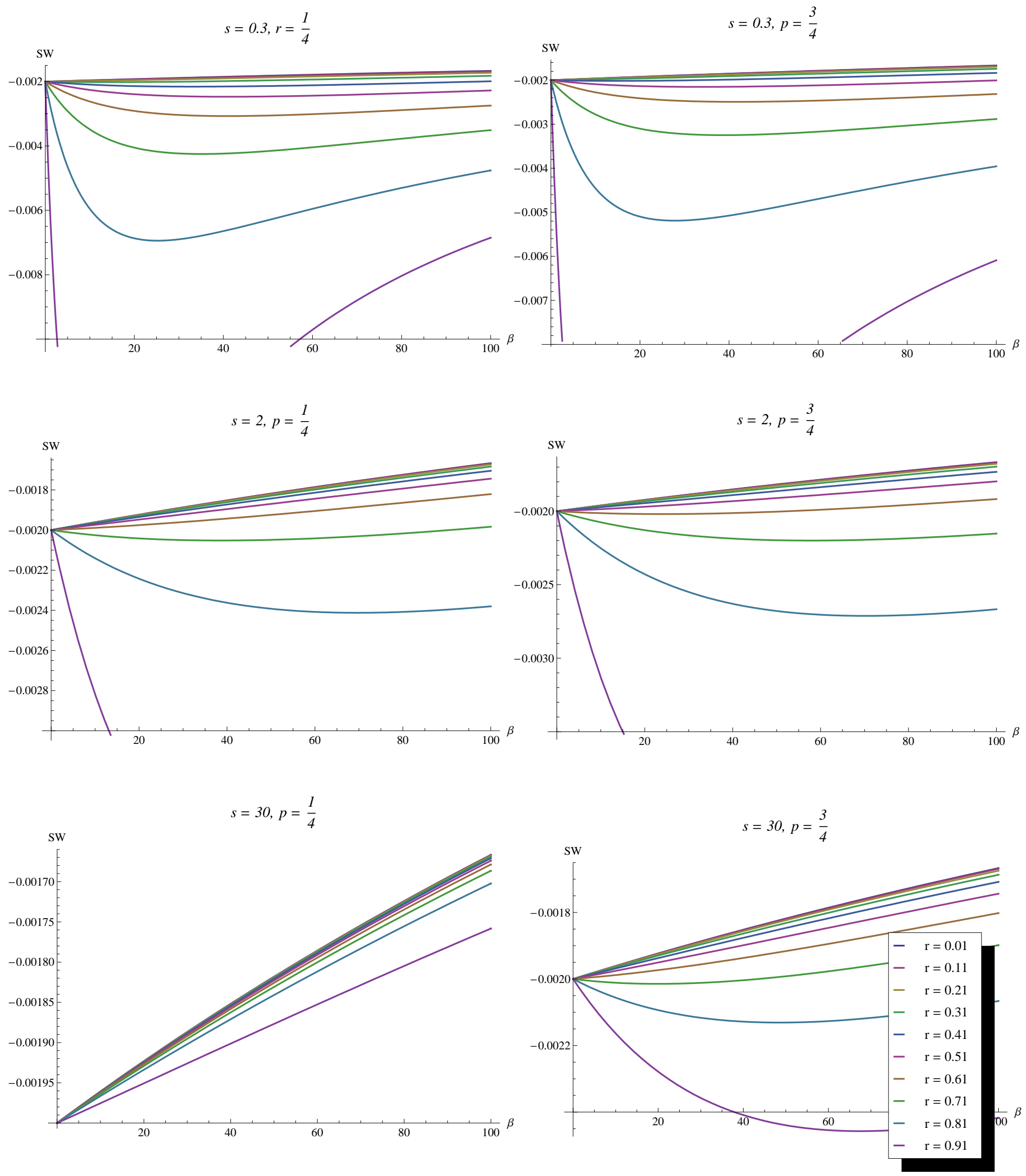

Figure 8: Proposition 10 Varying $r$ 

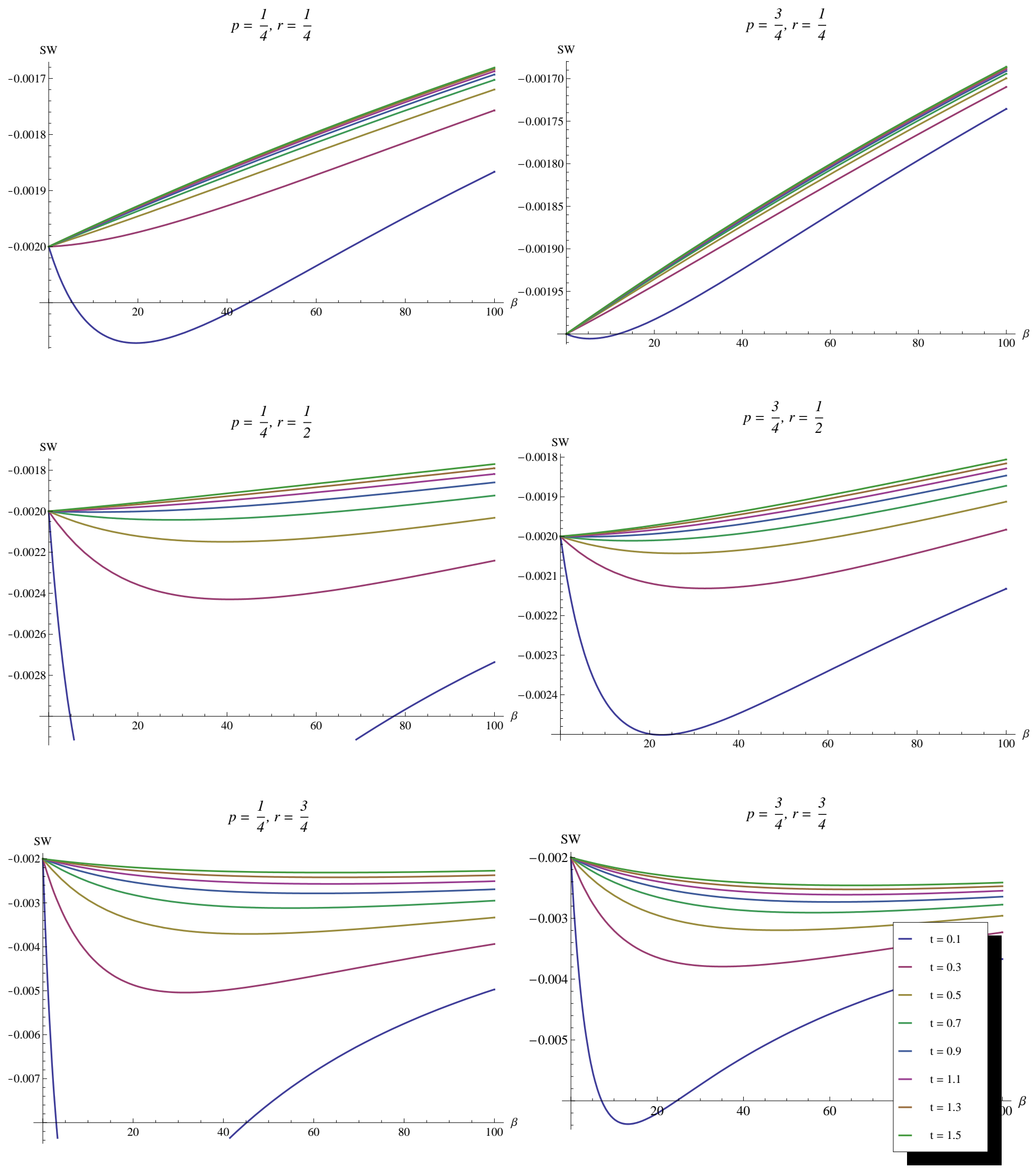

Figure 9: Proposition 10 (continued): Varying $t$ 

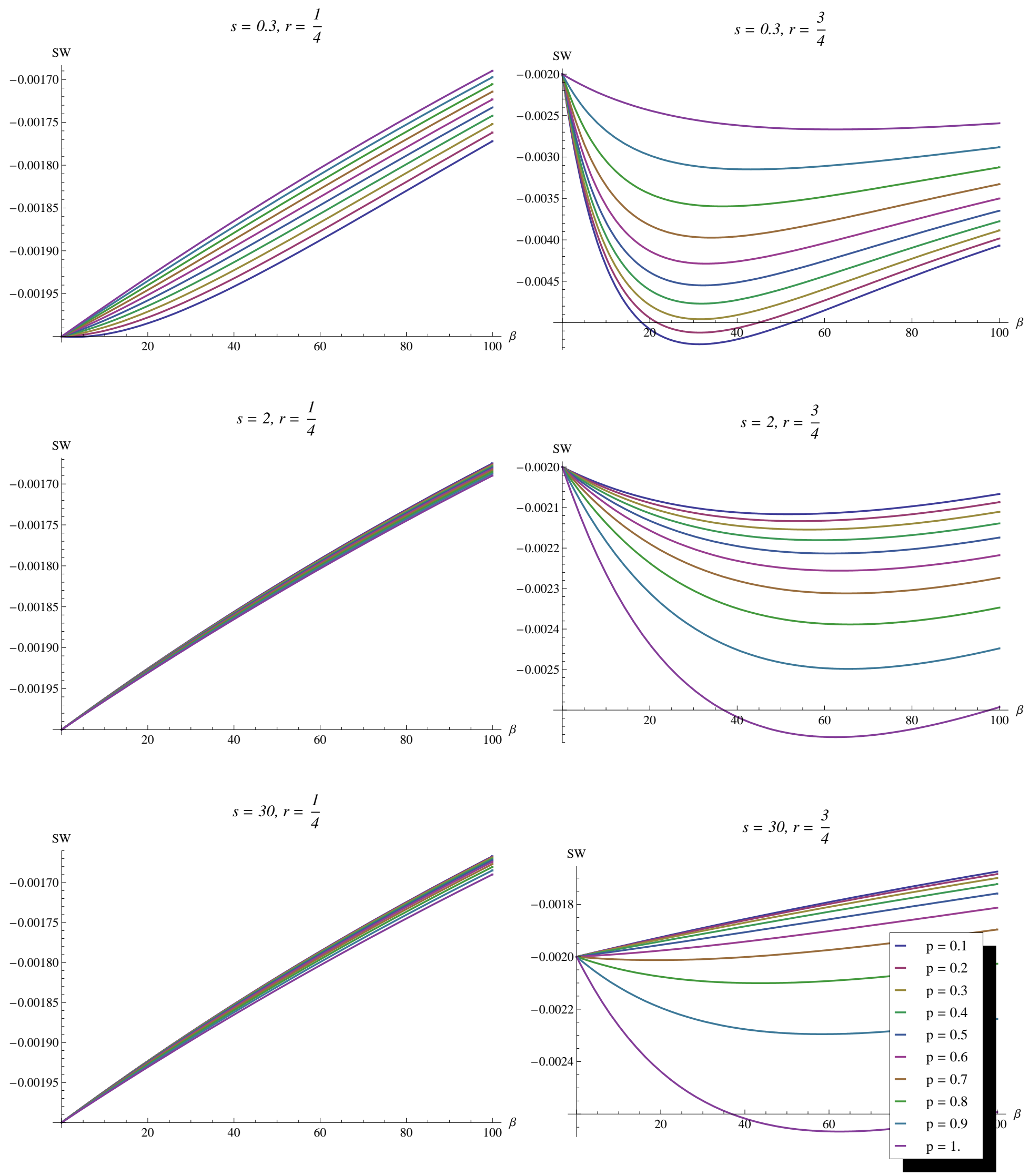

Figure 10: Proposition 10 (continued): Varying $p$ 